\title{
Instituto Superior Técnico Departamento de Engenharia Química
}

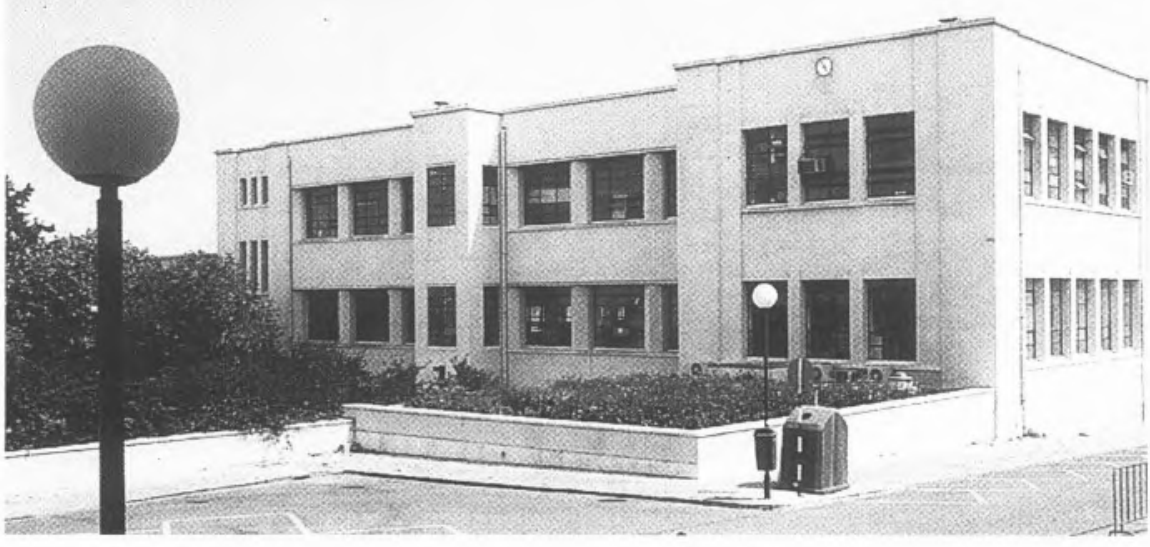

O Departamento de Engenharia Química (DEQ) do Instituto Superior Técnico tem uma grande tradição de ensino e investigação e orgulha-se de estar próximo de atingir o pleno de corpo docente doutorado (dos actuais 126 docentes cerca de 110 são doutorados) - possivelmente o primeiro grande departamento universitário a atingir tal meta. No entanto, em termos de espaços, o DEQ é actualmente o mais carenciado de todos os departamentos do IST, com o inconveniente acrescido de estar disperso por diversos edifícios (com realce para os Pavilhões de Química e de Minas, Complexo I e Instalações Piloto). A primeira prioridade da direcção do DEQ é resolver a curto e médio prazo os problemas de espaço e de segurança que daí advém. A aposta maior está, porém, na construção da Torre de Química, localizada entre os Pavilhões de Química e Minas.

\section{ESTRUTURA DO DEQ}

O orgão máximo do DEQ é o Conselho de Departamento (CDEQ), constituido por todos os professores e investigadores doutorados, por representantes eleitos dos assistentes, alunos e funcionários não docentes, e presidido pelo Presidente do Departamento. O CDEQ define as orientações gerais do Departamento, de âmbito científico e pedagógico. São ainda Orgãos de Gestão do DEQ, a Comissão Coordenadora, a Comissão Executiva e a Comissão Pedagógica do DEQ.
A Comissão Coordenadora do DEQ delibera sobre assuntos que não Química do IST

\section{$2^{\circ}$ ANO}

$1^{\circ}$ SEMESTRE

Análise Matemática III

Probabilidades e Estatística

Química Orgânica II

Química Analítica I

Electromagnetismo

Laboratório III

\section{$2^{\circ}$ SEMESTRE}

Análise Matemática IV

Química Inorgânica I

Química Orgânica III

Termodinâmica Química

Processos Químicos I

Laboratórios IV exijam a opinião directa de todos os membros do DEQ. Nela têm assento os membros da Comissão Executiva, o Coordenador de Licenciatura, o Presidente da Comissão Pedagógica do Departamento, o representante do DEQ à Comissão Coordenadora do Conselho Científico do IST, os Coordenadores das Secções do DEQ e o Director do Laboratório de Análises.

A gestão corrente do Departamento é assegurada pela Comissão Executiva, constituida pelo Presidente e Vice-Presidente do DEQ e por três a cinco vogais.

A Comissão Pedagógica tem como objectivo estimular o diálogo entre docentes e alunos e analisar e

Tabela I - Plano de Estudos do $1^{\circ}$ e $2^{\circ}$ Ano da Licenciatura em Engenharia

\begin{tabular}{|c|c|c|c|c|c|}
\hline & \multicolumn{3}{|c|}{ Horas / semana } & \multicolumn{2}{|c|}{ Total } \\
\hline & $\mathrm{T}$ & $\mathrm{P}$ & $\mathrm{L}$ & Horas & Créditos \\
\hline \multicolumn{6}{|l|}{$1^{\circ} \mathrm{ANO}$} \\
\hline \multicolumn{6}{|l|}{$1^{\circ}$ SEMESTRE } \\
\hline Análise Matemática I & 3 & 2 & & 5 & 4,4 \\
\hline Álgebra Linear & 3 & 2 & & 5 & 4,4 \\
\hline Química I & 4 & 1 & & 5 & 4,7 \\
\hline Programação & 2 & 1 & & 3 & 2,7 \\
\hline Int. Engenharia Química & 2 & 2 & & 4 & 3,4 \\
\hline Laboratório I & & & 4 & 4 & 1,6 \\
\hline Total & 14 & 8 & 4 & 26 & 21,2 \\
\hline \multicolumn{6}{|l|}{$2^{\circ}$ SEMESTRE } \\
\hline Análise Matemática II & 3 & 2 & & 5 & 4,4 \\
\hline Análise Numérica & 2 & 2 & & 4 & 3,4 \\
\hline Química II & 2 & & & 2 & 2,0 \\
\hline Mecânica Geral & 3 & 1 & 1 & 5 & 4,1 \\
\hline Química Orgânica I & 3 & 1 & & 4 & 3,7 \\
\hline \multirow[t]{2}{*}{ Laboratório II } & & & 6 & 6 & 2,4 \\
\hline & 13 & 6 & 7 & 26 & 20 \\
\hline
\end{tabular}

$\begin{array}{rrrrrr} & 3 & 2 & & 5 & 4,4 \\ & 3 & 2 & & 5 & 4,4 \\ & 3 & 1 & & 4 & 3,7 \\ & 2 & 1 & & 3 & 2,7 \\ & 3 & 1 & 1 & 5 & 4,1 \\ & & & 5 & 5 & 2,0 \\ \text { Total } & 14 & 7 & 6 & \mathbf{2 7} & \mathbf{2 1 , 3}\end{array}$

\begin{tabular}{|c|c|c|c|c|c|}
\hline & 3 & 2 & & 5 & 4,4 \\
\hline & 2 & 1 & & 3 & 2,7 \\
\hline & 3 & 1 & & 4 & 3,7 \\
\hline 1 & 3 & 2 & & 5 & 4,4 \\
\hline & 1 & 4 & & 5 & 3,8 \\
\hline & & & 4 & 4 & 1,6 \\
\hline Total & 12 & 10 & 4 & 26 & 20,6 \\
\hline
\end{tabular}



assento, para além o Presidente da Comissão Pedagógica do DEQ, os alunos representantes dos anos $\mathrm{e}$ ramos da licenciatura e os representantes docentes das Secções.

Existem presentemente no DEQ oito secções, reunindo cada uma delas docentes cuja actividade pedagógica e/ou científica se desenvolve dentro da mesma área da Química ou da Engenharia Química. A cada Secção, e em particular ao professor Coordenador, cabe pois a responsabilidade directa de um conjunto de disciplinas afins da licenciatura e de ensino pós-graduado, bem como da gestão do pessoal docente da Secção.

Para além dos orgãos deliberativos e excutivos enumerados, o DEQ conta ainda com várias comissões permanentes ou eventuais: Comissão de Informática, Comissão de Segurança e Obras, Conselho de Biblioteca e Conselho Consultivo. Este último orgão integra representantes da indústria, de laboratórios do Estado e de outras Universidades que, com a propor soluções para problemas de índole pedagógica. Neste Orgão têm

sua experiência, podem auxiliar o DEQ a definir orientações pedagógicas e científicas.

\section{Biblioteca do DEQ}

A Biblioteca do DEQ tem por objectivo fundamental o apoio às actividades de ensino e de Investigação, no âmbito das ciências básicas e aplicadas desenvolvidas no Departamento.

A Biblioteca organiza-se em três núcleos: o núcleo central, situado no piso térreo do Pavilhão de Engenharia Química, alberga cerca de 70 títulos periódicos e mais de 1000 não periódicos, cobrindo a generalidade das áreas científicas em que o Departamento se encontra envolvido. Estas instalações incluem salas de consulta, os serviçõs centrais de secretariado e apoio a utentes.

Os dois núcleos periféricos estão localizados nos dois últimos pisos do Pavilhão de Minas, especializando-se em electroquímica, corrosão e protecção de materiais (penúltimo piso) e em biotecnologia e tecnologia alimen-

Tabela II - Disciplinas do Tronco comum da Licenciatura em Engenharia Química do IST ( 3 e $4^{\circ}$ Anos)

\begin{tabular}{ccllll}
\multicolumn{3}{c}{ Horas / semana } & \multicolumn{3}{c}{ Total } \\
\hline $\mathrm{T}$ & $\mathrm{P}$ & $\mathrm{L}$ & Horas & Créditos \\
\hline
\end{tabular}

\section{$3^{\circ}$ ANO \\ $1 \%$ SEMESTRE}

Química Física I

Fenóm. de Transferência I

Processos Químicos II

Termodinâmica Química II

Métodos Computacionais

Laboratórios V

$\begin{array}{llll} & 3 & 1 & \\ \text { I II } & 3 & 2 & \\ & 1 & 4 & \\ \text { Total } & 2 & 2 & \\ & 2 & 2 & 4 \\ & 11 & 11 & 4\end{array}$

4
5
5
4
4
4
26

\section{SEMESTRE}

Química Física II

Fenóm. de Transferência II

Total

\begin{tabular}{ccccc} 
& 2 & 1 & 3 & 2,7 \\
& 3 & 1 & 4 & 3,7 \\
& 5 & 2 & 7 & $\mathbf{6 , 4}$ \\
\hline
\end{tabular}

\section{$4^{\circ} \mathrm{ANO}$}

\section{SEMESTRE}

Fenóm. de Transferência III

Engenharia das Reacções I

Processos de Separação I

Economia e Gestão*

Total

$\begin{array}{rrrr}3 & 2 & 5 & 4,4 \\ 2 & 1 & 3 & 2,7 \\ 2 & 3 & 5 & 4,1 \\ 2 & 1 & 3 & 2,7 \\ & & 16 & \mathbf{1 3 , 9}\end{array}$

- Funciona no $5^{\circ}$ Ano, $1^{\circ}$ Semestre, para os Ramos de Química Aplicada e Biotecnologia tar (último piso). Estes dois núcleos possuem salas de leitura, sendo facultados serviços de fotocópias, consulta e empréstimo de publicações.

Para além da constante actualização de recursos e existências, encontra-se actualmente em curso a sua informatização e ligação às restantes bibliotecas do IST. O sistema permitirá numa fase posterior o acesso a bases de informação bibliográfica localizadas no IST e a ligação a outras bibliotecas nacionais e estrangeiras.

\section{Laboratório de Análises}

O Laboratório de Análises, foi criado no final do século passado (1892) ainda no Instituto Industrial e Comercial de Lisboa, instituição que viria a dar origem ao Instituto Superior Técnico. Este laboratório, com grandes tradições de investigação e serviço no domínio da Análise Química, manteve sempre estreitas relações com o Departamento de Engenharia Química. De entre os químicos ilustres que foram seus directores destacam-se os nomes dos Professores Charles Lepierre, Herculano de Carvalho e Fraústo da Silva, todos eles docentes do IST.

Actualmente, o Laboratório de Análises é uma entidade autónoma que, em conjunto com as oito secções de ensino e investigação mais a biblioteca, é parte integrante do DEQ. A sua actividade de investigação aplicada e de serviço desenvolve-se predominantemente no domínio da análise química de águas e de controle de poluição através de análises de efluentes, rios e mar. Este laboratório de apoio e recurso para trabalhos de outras instituições e organismos oficiais, bem como de empresas privadas e de grupos de investigação, foi recentemente reequipado e modernizado, de modo a dar resposta às solicitações para estudos de carácter ambiental, verificação da carência ou de excesso de elementos em espécies animais ou vegetais, solos e águas e ainda à análise de elementos vestigiários em materiais de elevada pureza. 
Tabela III - Disciplinas da Especialidade no Ramo de Química Aplicada

\begin{tabular}{llll}
\multicolumn{4}{c}{ Horas / semana } \\
\hline $\mathrm{T}$ & $\mathrm{P}$ & $\mathrm{L}$ & Unidades de Crédito
\end{tabular}

\section{$3^{\circ} \mathrm{ANO}$}

$2^{\text {oSEM. }}$.

Mét. Instrument.de Análise I

Química Inorgânica II

Previsão de Propriedades

Laboratórios VI

1

Total

3

2

1

4

5

5

8

\section{$4^{\circ} \mathrm{ANO}$}

1 'EM.

Espectroscopia

Mét. Instrument.de Análise II

Laboratórios VII

\section{SEM.}

Processos Electroquímicos

Cinética Química

Processos Fotoquímicos

Química Analítica II

Mecanismos Reaccionais

Laboratórios VII

Total

total

\section{$5 \%$ ANO}

1 'sEM.

Química Orgânica Industrial

Análises Industriais e Controle

Controle de Poluição

2SEM.

Proj. Investigação Laboratorial

Opção

\section{ANUAL}

Projecto Químico Industrial

$$
\text { Total }
$$

1

\section{ENSINO}

\section{Licenciatura em Engenharia Química}

O ensino da Engenharia Química no IST é uma prática que acompanha o Instituto desde a sua fundação, caracterizando-se por oferecer uma formação sólida e simultaneamente inovadora.

A crescente complexidade dos problemas de engenharia química exige grande interdisciplinaridade $\mathrm{e}$ flexibilidade curricular para dar resposta aos novos desafios impostos pela constante mutação da indústria e da própria sociedade. Assim, em nome dessa flexibilidade, o Departamento de Engenharia Química do prático e industrial. Começando pela arquitectura molecular de novos compostos, passando pelo estudo das suas propriedades e reactividade, o processo culmina na exploração das suas aplicações potenciais. O ramo de Processos e Indústria privilegia a formação em ciências de engenharia e organiza-se segundo uma estrutura de engenharia de processo. Este ramo é o que segue mais de perto os cursos de engenharia química dos países anglo-saxónicos. Os alunos adquirem aqui conhecimentos adequados ao trabalho industrial (Engenheiro de Produção) e de projecto de equipamento (Engenheiro de Processos). O ramo de Biotecnologia alia à formação básica em química e em ciências de engenharia uma componente de ciências biológicas, dando particular relevo à tecnologia das fermentações e dos processos enzimáticos e à engenharia genética.

A escolha do ramo é feita a quando da inscrição no $3^{\circ}$ Ano, embora o primeiro semestre desse ano se inclua ainda no tronco comum do curso (Tabelas I e II), o que significa que as disciplinas orientadas para a especialização escolhida aparecem apenas no $6^{\circ}$ Semestre do curso.

Nos dois primeiros anos do curso, os alunos recebem uma formação básica em Matemática, Física e nas diversas áreas da Química (Inorgânica, Orgânica e Analítica). Os conhecimentos teóricos são complementados por trabalhos práticos em laboratórios autónomos semestrais (4 a $6 \mathrm{~h} / \mathrm{semana}$ ). Ainda no $1^{\circ}$ Ano, na disciplina de Introdução à Engenharia Química, os estudantes têm a sua primeira exposição à realidade industrial através de visitas de estudo e do estudo detalhado de uma indústria nacional que inclui o levantamento in loco do diagrama de fabrico e a descrição pormenorizada das peças de equipamento e das redes de utilidades. No $2^{\circ}$ Semestre do $2^{\circ}$ Ano, introduzem-se as ciências de engenharia, iniciando-se o ensino das disciplinas Termodinâmica Química e Processos Químicos. Nesta última, os estudantes integram os co- 
Tabela IV - Disciplinas da Especialidade no Ramo de Processos e Indústria

\begin{tabular}{|c|c|c|c|c|}
\hline & \multicolumn{3}{|c|}{ Horas / semana } & \multirow[b]{2}{*}{ Unidades de Crédito } \\
\hline & $\mathrm{T}$ & $\mathrm{P}$ & $\mathrm{L}$ & \\
\hline \multicolumn{5}{|l|}{$3^{\circ} \mathrm{ANO}$} \\
\hline \multicolumn{5}{|l|}{$2^{\circ}$ SEM. } \\
\hline Estratégia de Processos & 2 & 4 & & 4,8 \\
\hline Operações Sólido-Fluido & 2 & 1 & & 2,7 \\
\hline Mét. Instrument.de Análise & 3 & & & 3,0 \\
\hline Laboratórios VI & & & 8 & 3,2 \\
\hline Total & 7 & 5 & 8 & 13,7 \\
\hline \multicolumn{5}{|l|}{$4^{\circ} \mathrm{ANO}$} \\
\hline \\
\hline Materiais e Corrosão & 2 & 1 & & 2,7 \\
\hline Laboratórios VII & & & 6 & 2,4 \\
\hline \multicolumn{5}{|l|}{$2^{\circ}$ SEM. } \\
\hline Engenharia das Reacções ॥ & 2 & 1 & & 2,7 \\
\hline Optimização de Processos & 2 & 3 & & 4,1 \\
\hline Instrumentação e Controle de Processos & 2 & 2 & & 3,4 \\
\hline Práticas de Engenharia Química & & 3 & & 2,0 \\
\hline Processos de Separação II & 2 & 2 & & 3,4 \\
\hline Laboratórios VIII & & & 4 & 1,6 \\
\hline Total & 10 & 12 & 10 & 22,3 \\
\hline
\end{tabular}

\section{ANO}

1 SEM.

Engenharia das Reacções III

Instalações e Serviços Industriais

Controle de Qualidade

Complementos de Engenharia Química

$\begin{array}{llll}2 & 1 & 2 & 3,5 \\ 2 & 3 & & 4,1 \\ 2 & 3 & & 4,1 \\ & 4 & & 2,8\end{array}$

$2{ }^{\circ}$ SEM.

Projecto de Investigação Laboratorial

Opção

4

ANUAL

Projecto de Indústrias Químicas Total

nhecimentos de termodinâmica e estequiometria na quantificação de processos químicos simples, mediante técnicas de balanços de massa e de energia.

O $3^{\circ}$ ano do curso é dedicado ao ensino das ciências de engenharia (Química-Física, Termodinâmica Química e Fenómenos de Transferência), sendo a aprendizagem dos conceitos teóricos complementada por trabalhos laboratoriais integrados. As disciplinas do $1^{\circ}$ Semestre, são comuns aos três ramos da licenciatura; no $2^{\circ}$ Semestre deste ano, surgem, em cada ramo, as primeiras disciplinas da especialidade. No $4^{\circ}$ Ano do curso, acentua-se a distin-
Ramo de Biotecnologia (Tabela V), dá-se particular importância ao processamento de sistemas biológicos, pelo que o conjunto de conhecimentos científicos de engenharia química aqui ministrados reflectem a especificidade do tratamento daqueles sistemas.

O $1^{\circ}$ Semestre do $5^{\circ}$ Ano reflecte de forma acentuada a opção de ramo realizada, com disciplinas específicas que complementam a formação dos estudantes. Inicia-se simultaneamente a única disciplina anual da licenciatura, o Projecto, comum a todos os ramos, mas cuja temática respeita a especialização dos alunos. Esta disciplina, que impõe a realização de um ante-projecto completo de uma unidade fabril, constitui a cúpula da aprendizagem escolar e supõe elevado espírito de interdisciplinaridade, na medida em que recorre a grande parte dos conhecimentos adquiridos ao longo do curso para os utilizar de uma forma integrada.

No último reajuste curricular efectuado, procurou-se reduzir o número de disciplinas do $2^{\circ}$ Semestre do $5^{\circ}$ Ano, de modo a permitir aos estudantes finalistas concentrarem o esforço máximo realização do Projecto. Assim, do plano de estudo deste semestre consta adicionalmente uma Opção e o Projecto de Investigação Laboratorial. Nesta disciplina, os estudantes são iniciados na metodologia da investigação científica, realizando um projecto de investigação original, de pequena dimensão, sob a orientação de um professor do DEQ. Nalguns casos, é também aceite a realização deste projecto em laboratórios de investigação oficiais ou em universidades estrangeiras, frequentemente ao abrigo de programas de cooperação científicos.

Como disciplinas de opção, o DEQ oferece anualmente um leque variado de matérias (Tabela VI), impondo embora um número mínimo de pré-inscrições para o funcionamento das disciplinas. Em alternativa, é ainda dada a oportunidade aos estudantes de optarem por 
Tabela V - Disciplinas da Especialidade no Ramo de Biotecnologia

\begin{tabular}{|c|c|c|c|c|}
\hline & \multicolumn{3}{|c|}{ Horas / semana } & \multirow[b]{2}{*}{ Unidades de Crédito } \\
\hline & $\mathrm{T}$ & $\mathrm{P}$ & L & \\
\hline \multicolumn{5}{|l|}{$3^{\circ} \mathrm{ANO}$} \\
\hline \multicolumn{5}{|l|}{$2^{\circ}$ SEM. } \\
\hline Microbiologia & 3 & & & 3,0 \\
\hline Bioquímica & 3 & & & 3,0 \\
\hline Mét. Instrument.de Análise & 3 & & & 3,0 \\
\hline Laboratórios VI & & & 10 & 4,0 \\
\hline Total & 9 & & 10 & 13,0 \\
\hline \multicolumn{5}{|l|}{$4^{\circ} \mathrm{ANO}$} \\
\hline \multicolumn{5}{|l|}{$1^{\circ}$ SEM. } \\
\hline Tecnologia Microbiana & 3 & & & 3,0 \\
\hline Tecnologia Enzimática & 3 & & & 3,0 \\
\hline Laboratórios VII & & & 8 & 3,2 \\
\hline \multicolumn{5}{|l|}{$2^{\circ} \mathrm{SEM}$} \\
\hline Engenharia das Reacções II & 2 & 1 & & 2,7 \\
\hline Tecnologia de Fermentadores & 3 & & & 3,0 \\
\hline Instrumentação e Controle de Processos & 2 & 2 & & 3,4 \\
\hline Proc. Recuperação Produtos Biológicos & 3 & & & 3,0 \\
\hline Processos de Separação II & 2 & 2 & & 3,4 \\
\hline Laboratórios VIII & & & 8 & 3,2 \\
\hline Total & 18 & 5 & 16 & 27,9 \\
\hline
\end{tabular}

\section{$5^{\circ} \mathrm{ANO}$}

\section{$1 \stackrel{\circ}{\circ}$ EM.}

Tecnologia Alimentar

Instalações e Serviços Industriais

Tratamento de Efluentes

$\begin{array}{lll}2 & 2 & 3,4 \\ 2 & 3 & 4,1 \\ 3 & & 3,0\end{array}$

\section{$2^{\circ}$ SEM.}

Práticas de Engenharia Bioquímica

Opção

$\begin{array}{rrr} & 10 & 4,0 \\ 4 & & 2,8 \\ & & \\ 9 & & 9,3 \\ 18 & 10 & \mathbf{2 6 , 6}\end{array}$

uma qualquer disciplina de $2^{\circ} \mathrm{Se}$ mestre de um ramo diferente do seu.

\section{Regime de Acesso à Licenciatura}

Provas específicas: Matemática e Química ou, em alternativa, Matemática e Física.

Vagas (1994/95): 120 alunos

\section{Participação noutras Licenciaturas}

Para além de ser a entidade directamente responsável pela licenciatura em Engenharia Química do IST, o DEQ é ainda responsável pelo
Tabela VI - Disciplinas de Opção

Tecnologia Alimentar

Tratamento de Efluentes

Investigação Operacional

Refinação de Petróleos e Petroquímica

Métodos Radioquímicos

Polímeros

Tópicos de Biotecnologia

Gestão Industrial

Processos de Superfície

Segurança Industrial

Poluição

Química Organometálica

Biotecnologia

gia: Nestas três áreas, o IST atribuiu, entre 1986 e 1993, 112 graus de Mestre em Engenharia Química. O DEQ participa ainda no Mestrado em Ciências dos Alimentos da UTL, em colaboração com outras Escolas desta Univesidade (ESMV-ISA-ISE-IST).

Em simultâneo com os cursos de Mestrado, existem programas de doutoramento, em áreas científicas variadas: Química Teórica, Termodinâmica Química, Processos de Separação, Engenharia Biológica, etc. Como indicador do dinamismo destes programas podemos referir que no período 198694 o IST concedeu o grau de Doutor em Engenharia Química a cerca de 130 doutorandos, entre assistentes e bolseiros sem vínculo à Escola.

\section{Investigação}

A tradição de investigação no IST no domínio da Química remonta ao tempo do Prof. Charles Lepierre, químico notável cujos trabalhos de investigação sobre a análise e estudo dos equilíbrios físico-químicos de águas naturais, fizeram escola na Universidade Portuguesa.

Actualmente, a quase totalidade dos docentes do DEQ desenvolve os seus projectos de investigação integrados no plano de actividades científicas de Institutos de Investigação (Instituto de Biotecnologia e Química Fina) e em 4 Centros de Investigação do IST (Centro de Química Estrutural, Centro de Engenharia Biológica e Química, Centro de Processos Químicos e Centro de QuímicaFísica Molecular). 